\title{
Design of a weak fluorescence detection circuit in molecular diagnosis
}

\author{
Huajuan $\mathrm{BAI}^{\mathrm{a}}$, Jihong $\mathrm{FENG}^{\mathrm{b}}$, and Hong ZHANG ${ }^{\mathrm{c}}$ \\ Beijing University of technology, Beijing 100124, CHINA \\ abaihuajuan@emails.bjut.edu.cn, bjhfeng@bjut.edu.cn
}

Keywords: Molecular diagnosis, weak fluorescence detection, photomultiplier tubes

\begin{abstract}
Fluorescence is generally adopted in molecular diagnosis. In order to detect weak fluorescence signal, a weak fluorescence detection circuit has been design based on a photomultiplier tube. The circuit mainly consists of three parts which are a photoelectric converter circuit, a pre-amplifier circuit and a filter circuit. This circuit is able to receive weak fluorescent signals and convert them into electrical signals effectively. As a result, important information such as the concentration of corresponding reagnet can be obtained by analyzing the intensity of fluorescence received. It is experimentally demonstrated that the designed circuit can be used in weak fluorescence detecting efficiently. Its detection limitation is as low as the fluorescent signal emitted from quantum dots of $10^{-10} \mathrm{~mol} / \mathrm{ml}$, making it suitable for low fluorescence detection in molecular diagnosis.
\end{abstract}

\section{Introduction}

Molecular diagnosis is an important frontier area in modern medicine. It pushes the detection of the disease moving from tissue and cellular level up to the molecular level, but also greatly reduces the detection time. Its core is a specific genetic diagnosis which main technology consists of nucleic acid hybridization, polymerase chain reaction and biochip technology [1]. Molecular diagnostic techniques give rise to a new revolution for life science research, making the signal detection from the radionuclide marker into a fluorescent marker, obtaining the reagents concentration and other important information of corresponding compounds by analyzing the fluorescence intensity [2, 3]. Therefore, photoelectric detection technology will determine the level of molecular diagnostic techniques

Photoelectric detection technique is used certain illuminated substances emitting out the fluorescent light which are able to reflect the material properties to do qualitative or quantitative analysis. However, since the excited fluorescence generally has a low intensity and short fluorescence lifetime or a low fluorescence efficiency, a weak fluorescence detection circuit with practical and reasonable low noise, high signal to noise ratio to extract the weak optical signal from the noise background $[4,5]$. Photoelectric detection technology has its application in many areas and a variety of detection methods are developed, but due to the limited sensitivity of the commonly used methods, it is difficult to meet the requirements [6]. In this paper, photoelectric detection include the photoelectric converting circuit using a low noise, fast response, large reception area photomultiplier tube, a preamplifier circuit using the OPA4277, a filtering circuit using a low-pass second-order active filter. The method has high accuracy, good stability which is applied a high-precision optical detector and a high-performance operational amplifier.

\section{Weak fluorescence detection}

In the weak fluorescence detection, the fluorescent signal is generally very weak and often buried in noise. Therefore, the weak fluorescence detection is typically needed to extract from noise, to be amplified, to be filtered, and then to be transmitted into a digital signal through $\mathrm{d} / \mathrm{a}$ converter to subsequent processor circuits. This photoelectric detection system is composed of a photomultiplier tube (PMT), preamplifier circuit, filter circuit, A/D conversion circuit, DSP control and signal processing circuit and so on, its structure diagram shown in figure 1. 


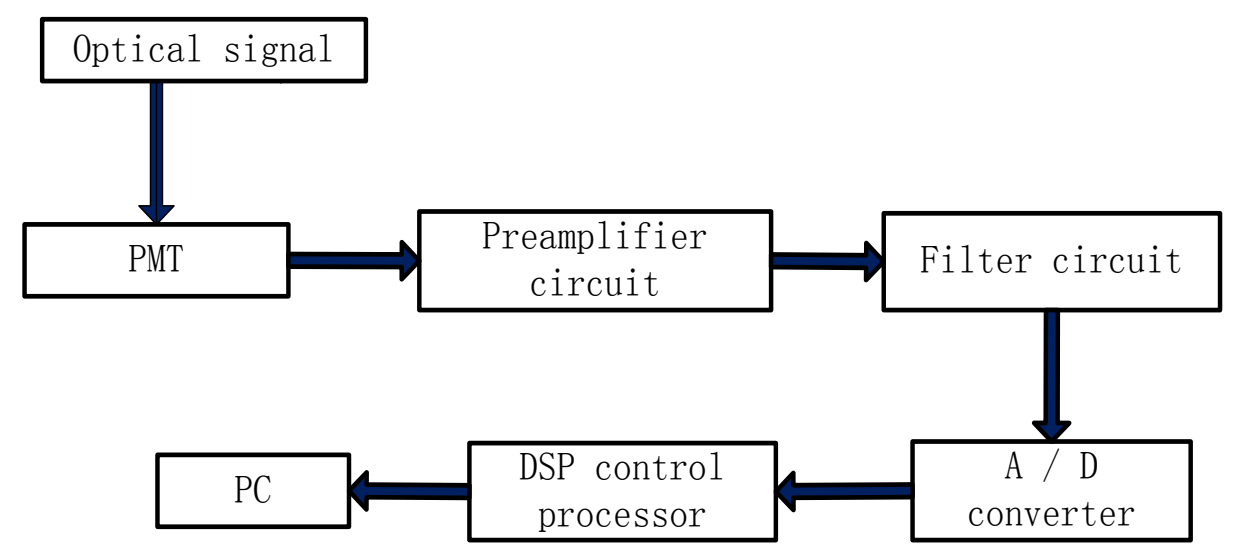

Fig. 1. Weak fluorescence detection system diagram

\section{Design of a circuit for weak fluorescence detection}

Photoelectric conversion circuit. The photoelectric sensor is the key device in the photoelectric conversion circuit because its sensitivity and dynamic response speed is the speed and precision of the detection circuit's work prerequisites [7]. In all kinds of light sensor, a PMT is one of the best performances which are well ahead of other light sensors among the coefficient of sensitivity, response speed, noise and dynamic range. It is more valuable that it outputs signal in large range with a high degree of linear output [8, 9]. A high-performance PMT is used in this study. In the PMT output, a reverse proportion op-amp circuit is connected to which makes the output of the PMT module converting into the forward voltage signal, convenient for subsequent signal processing.

In this paper, the photomultiplier tube control circuit is designed. The circuit is powered by +12 voltage supply which is first converted to +5 voltage by a LM2596, again converted to +15 voltage by a DCP020515, and finally regulated to about +13 volt output by a zener diode. At the same time, a sensitivity adjustment module is designed in the circuit which can easily adjust the sensitivity of PMT through adjust potentiometer knob.

Pre-amplification electric circuit. Low noise preamplifier which performance directly affects the precision of the system is a critical part of the whole system. In weak signal detection system, the requirement for the preamplifier is particularly strict. First, the preamplifier should have strong anti-interference ability, and through the low noise circuit design makes its noise is far less than the measured signal. Second, the preamplifier in wide frequency range should have enough gain with high stability. Therefore, in the design to reduce the preamplifier's noise coefficient, one of the most important is the reasonable choice and use of the low noise operational amplifier.

In this paper, an OPA4277 is selected to structure the amplifying circuit. At the same time, cooperating with the operational amplifier, the resistor requires noise coefficient, small error, good voltage stability, and so on. We choose precision metal film resistors which precision is $0.1 \%$ to meet the requirements of circuit design. To ensure the PMT has good linear and frequency response characteristics it is needed to make its load resistance large. Load resistance is too small to affect the output response efficiency. If an operational amplifier is used to replace the load resistance, the output efficiency can solve. Here an operational amplifier OPA4277 is adopted instead of load resistance and the current feedback is used to compensate dc part of dark current.

Filter circuit. Since the detected fluorescence signal frequency is low commonly in molecular diagnosis, a second-order active low-pass filter circuit is designed in order to filter out high frequency interference of the voltage signal which photoelectric detection system outputs. Furthermore to reduce the power frequency $50 \mathrm{~Hz}$ and higher harmonic component's interference, the filter cutoff frequency is designed to be $\mathrm{f}_{0}=40 \mathrm{~Hz}$. The second-order low-pass filter circuit transfer function expression is as below. 


$$
A(s)=\frac{V_{n}(s)}{V_{i}(s)}=\frac{A_{V F}}{1+\left(3-A_{V F}\right) s C R+(s C R)^{2}}
$$

Here, $A(s)$ is a second-order low-pass filter circuit transfer function, $V_{n}(s)$ is the output function, $V_{i}(s)$ in the input function, $A_{V F}=A_{0}$ is pass band gain, filter quality factor $Q=1 /\left(3-A_{V F}\right)$. When $A_{V F}$ is less than 3 , the circuit can stably work. When the $A_{V F}$ is more than 3 , the circuit will self-excited oscillate. Using software Multisim to simulate, the circuit diagram is shown in figure 2 and the simulation result is shown in figure 3.

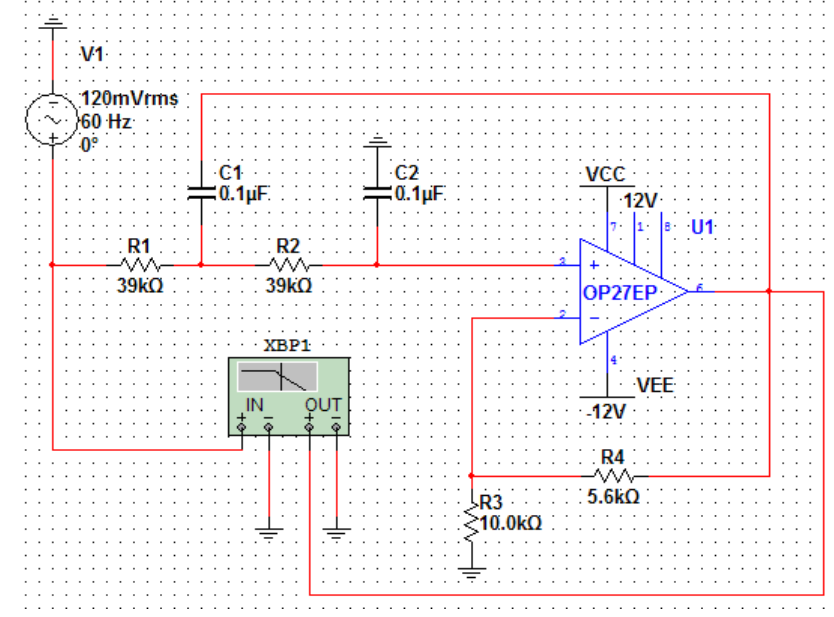

Fig. 2. Circuit in Multisim simulation

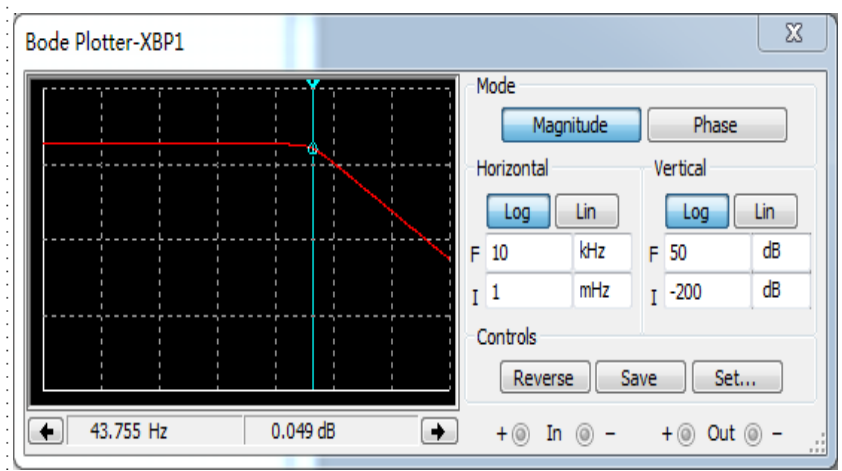

Fig. 3. Simulation result for circuit

In Fig. 3, the horizontal ordinate is the second-order low-pass filter circuit frequency f/Hz while the vertical ordinate is the voltage gain $20 \lg \mathrm{A} / \mathrm{dB}$. It is shown that the filter circuit voltage gain vary as the input frequency change. It can be seen that $20 \lg \mathrm{A}_{0}$ is about equal to $3.8 \mathrm{~dB}$ and the cut-off frequency of filter $\mathrm{f}_{0}$ is about equal to $44 \mathrm{~Hz}$. There is a little deviation with design requirements. This is mainly due to there are deviations in the resistances and the capacitance; however, it is within the range of allowable error. When $\mathrm{f}>\mathrm{f}_{0}$, the curve reduces with $40 \mathrm{db} /$ ten octave attenuation. Therefore, when the $\mathrm{f}_{0}=4 \mathrm{k} \mathrm{Hz}$, the dc gain drop from 3.8dB down to about $76 \mathrm{~dB}$.

\section{System test}

At present, there is no weak fluorescence determination of technical specifications in domestic. With reference to the American association of Materials and test (American Society for Testing and Materials, ASTM) and the National Institute of Standards and Technology (National Institute of Standards and Technology, NIST) issued for fluorescence measurement in the field of biomedical technology standard norms, some basic index of this system are measured $[10,11]$.

According to the recommended of ASTM E578-01 determination for the fluorescence detection sensitivity, we choose Rhodamine B methanol solution with the biggest fluorescence wavelength of $610 \mathrm{~nm}$ as a liquid for fluorescent detection. The reagent is processed with serial 2-fold dilution from its original concentration of $7.5^{\prime} 10^{-10} \mathrm{~mol} / \mathrm{ml}$. In order to increase accuracy, we repeated 5 times to detect the same concentration of reagent in our experiment. The average value of the fluorescence 5 times intensities at the same concentration works as the final value. In photoelectric detection system, the photomultiplier gain control voltage is set 0.85 voltages. Assembling a bandpass filter with its bandpass width of $35 \mathrm{~nm}$ for a quantum dot test, the fluorescent test curve is shown in figure 4 . 


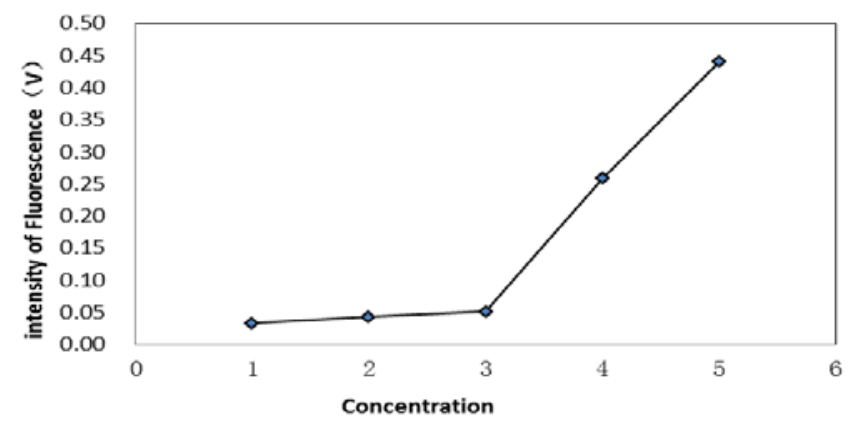

Fig. 4 Fluorescence detection curve of quantum dots

\section{Discussion}

Analysis of above test curve, it is seen that the designed circuit can detect the minimum concentration of at least $1.50 \times 10^{-10} \mathrm{~mol} / \mathrm{ml}$ for the quantum dot which emits the fluorescent light with the center wavelength at 610 . Within the scope of the testing, by the experimental data we can calculate the R2 $=0.9982$. It is experimentally demonstrated that the designed circuit with linearity can effectively detect the weak fluorescence and is suitable for the molecular diagnosis.

\section{References}

[1] WU Zhiyuan, ZHANG Chen, GUAN Ming. Evolutions in half a century: the course of change and development of current molecular diagnostics techniques. Laboratory Medicine, March 2014

[2] HUANG Shan. The application of molecular diagnostic techniques in the diagnosis and teveatment of cardiovascular disease. Journal of molecular diagnostics and therapy, 2014(6): 279-282.

[3] SONG Tao, ZAHNG Bin, LUO Qianqian. Photoelectric Conversion Circuit Design and Optimization. Electro-optic Technology Application, 2010(6): 46-48

[4] WANG Li-gang, ZHANG Dianyuan. Research and design of photoelectric detection circuit with low noise. Electrical Measurement\&Instrumentation. 2007, 44(500): 63-66.

[5] WANG Meng, LIU Jia, MIN Li. Design of photoelectric detection circuit of weak signal and noise analysis. Electronic Design Engineering, 2014

[6] Zeng Qingyong. Weak signal detection ( Zhejiang University Press, Hangzhou, 2004).

[7] WANG Hongling, Hu xidong, Chen Wei, Tang Bing. The Application of Photon counter PMT in Fluorescence Check and Measure System. Microcomputer Information, 2005, 21(002): 144-145.

[8] CHENG Jinxing, MAO Yongze, ZHANG Anhui. Study on Photomultiplier response characteristics of dozens of ms pulse width signal level. Nuclear Electronics \& Detection Technology, 2007, 27(3): 436-438.

[9] SON Fujia, CHEN Shuoshuo, CAI Jinda, YOU Qianlin. Development of Chemiluminesecence Immune Detection System Based on Photomultiplier Tube. Chinese Journal of Biomedical Engineering, 2012, 21(6): 859-865

[10]P.C. DeRose. Recommendations and Guidelines for Standardization of Fluorescence Spectroscopy, NISTIR 7457 National Institute of Standards and Technology, Gaithersburg, MD, 2007.

[11] Armbruster D. A., Pry T.I., Limit of Blank, Limit of Detection and Limit of Quantitation. Clin. Biochem. Rev, 2008, 29(1): S49-S52. 